\title{
Energy Efficient Resource Allocation in 5G Hybrid Heterogeneous Networks: A Game Theoretic Approach
}

\author{
Hamnah Munir*, Syed Ali Hassan*, Haris Pervaiz ${ }^{\dagger}$, Qiang $\mathrm{Ni}^{\dagger}$ and Leila Musavian ${ }^{\dagger}$ \\ ${ }^{*}$ School of Electrical Engineering \& Computer Science (SEECS), National University of Sciences \& Technology \\ (NUST), Islamabad, Pakistan \{14mseehmunir, ali.hassan\}@ seecs.edu.pk \\ ${ }^{\dagger}$ School of Computing \& Communications, Lancaster University, UK. \\ \{h.pervaiz, q.ni, 1.musavian\}@lancaster.ac.uk
}

\begin{abstract}
Millimeter wave (mmWave) technology integrated with heterogeneous networks (HetNets) has emerged as a new wave to overcome the thirst for higher data rates with low transmission powers and severe shortage of spectrum in the wireless industry. In this paper, we consider the uplink of a hybrid heterogeneous network with femtocells overlaid on a macrocell, and formulate a two layer game theoretic framework to maximise the energy efficiency while optimising the network resources. The outer layer non-cooperative game-theoretic approach allows each femtocell access point (FAP) to maximise the data rate of its users by selecting the frequency band either from the sub$6 \mathrm{GHz}$ and the mmWave. The solution to the non-cooperative game can be obtained by using pure strategy Nash equilibrium (PSNE). The inner layer game-theoretic approach ensures the energy efficient user association method subject to the minimum rate and maximum transmission power constraints by using Lagrangian dual decomposition approach. Simulation results show that the proposed hybrid HetNet scheme exploiting the mmWave frequency band improves the sum-rate and energy efficiency in comparison to the scenario where all the networks operate at sub-6 $\mathrm{GHz}$ frequency band. The performance of the hybrid HetNet scheme can be further enhanced by incorporating the power control mechanism.
\end{abstract}

Index Terms-Heterogeneous network, millimeter wave, game theory, energy efficiency, sum-rate, hybrid network.

\section{INTRODUCTION}

To manage the staggering growth of wireless data traffic, HetNets have drawn tremendous attention in the next generation wireless communication systems. Heterogeneity in the wireless environment allows low power base stations (BSs), deployed in small cells of diverse sizes overlaid on a macrocell, to operate at different frequency bands that makes an efficient use of the radio resources [1]-[3].

Millimeter wave (mmWave) technology has gained significant attention in the race of effective solutions to $5 \mathrm{G}$ requirements [4]-[6]. The spectrum available in mmWave bands, ranging from $10-300 \mathrm{GHz}$, is many times wider than the existing cellular networks. While improving network performance, it faces many challenges including hardware expenses, non-line-of-sight (NLOS) signal range and large distance connections. However, with the help of highly directional antennas and beamforming, significant signal strength can be achieved within a range of about 150-200 meters. Significant advancements have also been seen in the manufacturing of low cost mmWave hardware [7]. The coexistence of noise limited mmWave small cells with interference limited conventional UHF overlay in a hybrid heterogeneous network will resolve the hardware problem along with improvement in the overall network performance.

This flexibly of air-interfaces and increasing network scalability has made the centralized control a challenging task. In this regard, user-centric schemes have emerged as potential solutions to overcome the complexity of centralized monitoring by authorizing users to make decisions with or without network assistance at less computational complexity. The fusion of user-centric approach, which focuses on the interest of users, and network-centric approach, which focuses on the interest of network, can generate interesting results [8]. Another concern aggravated due to the drastic increase in the data traffic and substantial growth of network infrastructures is energy consumption [9]. This challenge has made developing energy efficient system, a key necessity for the next generation mobile networks. HetNets, consisting of small cells with smaller coverage range, allows BSs and user equipments (UEs) to communicate at lower powers which results in the reduction of energy consumption and also the interference [10], [11].

In this paper, we formulate a two layer framework for energy efficient resource allocation in a hybrid heterogeneous network. In the first game, each femtocell access point (FAP) models its preferred access policy for both mmWave and UHF frequency bands, given the state of the network, to optimise the data rates of its home users. Then, these FAPs opt for one of these bands in the best interest of the network using a network-centric approach. To solve this game, we devise a scheme, which always reaches a pure strategy Nash equilibrium (PSNE). It is then followed by the next game where MUEs finalize their association while maximizing energy efficiency (EE) considering the power and minimum rate constraints. This is done in a user-centric fashion with network assistance. This game is solved using Lagrangian dual decomposition approach. The performance of this hybrid HetNet is compared with the stand alone UHF networks.

The rest of this paper is organized as follows. In Section 
II, we present the system model of the proposed framework. In Section III, we discuss the game formulation. Section IV shows the simulation results and Section $\mathrm{V}$ concludes the paper.

\section{System Model}

Consider the uplink of a two-tier heterogeneous network (HetNet) having $M$ femtocell access points (FAPs) overlaid on a macrocell, as shown in Fig. 1, where a total of $N$ macrocell user equipments (MUEs) are randomly distributed. Let $M=$ $M_{M} \cup M_{\mathbb{U}}$ be the set of FAPs where $M_{M}$ represents the set of FAPs operating on mmWave band and $M_{\Perp}$ be the set of FAPs operating on UHF band whereas $M_{\odot}=\left\{m_{0}\right\}$ be the singleton set representing macrocell base station (MBS). Similarly, let $\mathbb{N}=\mathbb{N}_{\odot} \cup \mathbb{N}_{M}$ be the set of MUEs where $\mathbb{N}_{\odot}$ be the set of MUEs connected to MBS and $\mathbb{N}_{M}=\bigcup_{m=1}^{M} \mathbb{N}_{m}$ be the set of MUEs connected to the $m^{t h}$ FAP. On the other hand, $\mathbb{F}=\bigcup_{m=1}^{M} \mathbb{F}_{m}$ denotes the set of femtocell user equipments (FUEs) where each $\mathbb{F}_{\mathrm{m}}=\{1,2, \ldots, F\}$ is the set of FUEs connected to a single FAP. Also let $\mathbb{\square}=\mathbb{N} \cup \mathbb{F}$ be the set of all the users in the network and $\mathbb{J}=M \cup \mathbb{N}_{\odot}$ be the set of all the base stations in the network.

The bandwidth allocated to each FAP depends on the type of communication link, i.e., mmWave or UHF. The FAPs operating on mmWave band split the bandwidth, $B_{m}$, into identical $K_{m}$ sub-bands depending on the number of users connected to them. On the other hand, FAPs operating on conventional UHF bands assign the entire frequency band $B$ consisting of $K$ subcarriers to all the connected users. The same bandwidth, $B$, is also used by the MBS, which is assumed to operate on the UHF band. Hence each MUE gets bandwidth $B$ comprising of $L$ subcarriers, which introduces cross-tier interference between the femtocells operating on UHF and the macrocell. The path loss models for this system are expressed by the following equations for mmWave and UHF links, respectively

$$
\begin{gathered}
L_{\mathrm{mmW}}(d)[\mathrm{dB}]=\left\{\begin{array}{lr}
b+10 \alpha_{L} \log (d)+\Omega_{L} & \text { if link is LoS } \\
b+10 \alpha_{N} \log (d)+\Omega_{N} & \text { otherwise. }
\end{array}\right. \\
L_{\mathrm{UHF}}(d)[\mathrm{dB}]=20 \log \left(\frac{4 \pi}{\lambda_{c}}\right)+10 \beta \log (d)+\Psi,
\end{gathered}
$$

where $d$ is the distance in meters, $\Omega_{L}$ and $\Omega_{N}$ are zero mean $\log$ normal random variables for line-of-sight $(\mathrm{LoS})$ and nonline-of-sight (NLoS) mmWave links, respectively. $\Psi$ represents the log normal random variable in the case of UHF links.

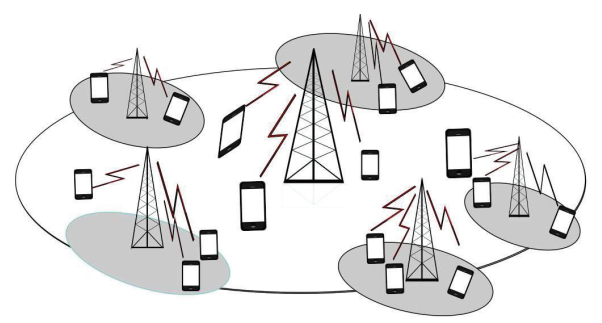

Figure 1. A heterogeneous network with femtocells overlaid on a macrocell
In (1), $b=32.4+20 \log \left(f_{c}\right)$ shows the fixed path loss for mmWave links, where $f_{c}$ is the carrier frequency. Similarly in (2), $\lambda_{c}$ corresponds to the carrier wavelength in case of UHF link. The path loss exponents for LoS and NLoS mmWave links are indicated by $\alpha_{L}$ and $\alpha_{N}$, respectively, whereas the path loss exponent for UHF links is denoted by $\beta$.

To maintain the quality-of-service $(\mathrm{QoS})$ requirements of the users, a constraint on the cross-tier interference is applied to find the optimal transmit power of the users. Let $\rho_{i}^{j}[x] \epsilon$ $\{0,1\}$ denotes the connection between any $i^{\text {th }}$ user and any $j^{\text {th }} \mathrm{BS}$ on any subcarrier $x$. In case of connectivity, $\rho_{i}^{j}[x]=$ 1 , otherwise $\rho_{i}^{j}[x]=0$. Let $I_{\mathrm{MBS}}[l]$ denote the interference threshold for the MBS on the $l^{\text {th }}$ subcarrier and we have

$$
\sum_{j \in \mathbb{M}_{\mathrm{U}}} \sum_{i \in \mathbb{F}_{\mathrm{j}} \cup \mathbb{N}_{\mathrm{j}}} \rho_{i}^{j}[l] g_{i j}[l] p_{i}^{j}[l] \leq I_{\mathrm{MBS}}[l],
$$

where $g_{i j}$ is product of the magnitude squared of the channel gain and the inverse of the path loss between the $i^{t h}$ user and the $j^{\text {th }} \mathrm{BS}$ and $p_{i}^{j}$ represents the optimal transmit power of the $i^{\text {th }}$ user with the constraint that

$$
p_{i} \leq P_{i}^{\max } \quad \forall i,
$$

where $p_{i}^{\max }$ is the maximum allowable transmit power for the $i^{\text {th }}$ user.

Similarly, $I_{\mathrm{U}(m)}[k]$ denotes the interference threshold on the $k^{t h}$ subcarrier for the $m^{t h}$ FAP operating on UHF band and the condition becomes

$$
\sum_{\substack{j \in M_{\cup} \cup M_{o} \\ j \neq m}} \sum_{i \in \mathbb{F}_{j} \cup \mathbb{N}_{j}} \rho_{i}^{j}[k] g_{i j}[k] p_{i}^{j}[k] \leq I_{\mathrm{U}(m)}[k],
$$

and $I_{\mathrm{M}(m)}[k]$ denotes the interference threshold on the $k_{m}^{t h}$ subcarrier for the $m^{\text {th }}$ FAP operating on mmWave band and the constraint becomes

$$
\sum_{\substack{j \in \mathbb{M}_{\mathrm{M}} \\ j \neq m}} \sum_{i \in \mathbb{F}_{j} \cup \mathbb{N}_{\mathrm{j}}} \rho_{i}^{j}\left[k_{m}\right] g_{i j}\left[k_{m}\right] p_{i}^{j}\left[k_{m}\right] \leq I_{\mathrm{M}(m)}\left[k_{m}\right] .
$$

The transmit power of the $i^{\text {th }}$ user at the $j^{\text {th }} \mathrm{BS}$, separated by the distance $d_{i j}$, is represented by $p_{i}^{j}$. The channel between them on subcarrier $x$ is represented by $h_{i j}[x]$. The received power from any $i^{\text {th }}$ user at any $j^{\text {th }}$ BS on subcarrier $x$ is given as

$$
\mu_{i}^{j}[x]=\left\{\begin{array}{lr}
\frac{p_{i}^{j} G\left(\theta_{j}\right)\left|h_{i j}[x]\right|^{2}}{L_{\mathrm{mmw}}\left(d_{i j}\right)} & \text { mmWave, } \\
\frac{p_{i}^{j}\left|h_{i j}[x]\right|^{2}}{L_{\mathrm{UHF}}\left(d_{i j}\right)} & \mathrm{UHF},
\end{array}\right.
$$

where $G($.$) represents the antenna gain and \theta_{j}$ is the azimuthal angles of BS beam alignment. Here, a sectored approximation to the beam pattern is assumed. If $\theta \in\left[\theta_{0}-\frac{\Delta \omega}{2}, \theta_{0}+\frac{\Delta \omega}{2}\right]$, where $\Delta \omega$ is the half power beamwidth, then the perfect alignment of the transmitter beam is considered and its gain is denoted by $G_{\max }$. The gain, in case of a misaligned beam, is $G_{\min }$. The channel gain $h$ follows Rayleigh or Rician distribution for LoS or NLoS links, respectively.

The signal-to-interference plus noise ratio (SINR) of the $f^{t h}$ FUE on subcarrier $k_{m}$ at the $m^{t h}$ FAP operating on 
mmWave band is given by

$$
\operatorname{SINR}_{f}^{m}\left[k_{m}\right]=\frac{\mu_{f}^{m}\left[k_{m}\right]}{\sigma^{2}\left[k_{m}\right]+I_{f}^{m}\left[k_{m}\right]},
$$

where $I_{f}^{m}\left[k_{m}\right]$ is defined by

$$
\begin{array}{r}
I_{f}^{m}\left[k_{m}\right]=\sum_{\substack{j=1 \\
j \neq m}}^{M} \sum_{i=1}^{F}\left(1-\prod_{\substack{a=1 \\
a \neq m}}^{\mathbb{M}_{\mathbf{M}}} \mathbb{1}_{\rho_{i_{j}}^{a}\left[k_{m}\right]=0}\right) \mu_{f}^{m}\left[k_{m}\right]+ \\
\sum_{n=1}^{N}\left(1-\prod_{\substack{j=1 \\
j \neq m}}^{\mathbb{M}_{\mathrm{M}}} \mathbb{1}_{\rho_{n}^{j}\left[k_{m}\right]=0}\right) \mu_{n}^{m}\left[k_{m}\right],
\end{array}
$$

whereas the SINR of the $f^{\text {th }}$ FUE on subcarrier $k$ at the $m^{\text {th }}$ FAP operating on UHF band is given by

$$
\operatorname{SINR}_{f}^{m}[k]=\frac{\mu_{f}^{m}[k]}{\sigma^{2}[k]+I_{f}^{m}[k]},
$$

where $I_{f}^{m}[k]$ is defined by

$$
\begin{array}{r}
I_{f}^{m}[k]=\sum_{j=1}^{M} \sum_{\substack{i=1 \\
(i \neq f \\
\text { when } j=m)}}^{F}\left(1-\prod_{a=1}^{M_{\mathrm{U}}} \mathbb{1}_{\rho_{i_{j}}^{a}[k]=0}\right) \mu_{f}^{m}[k]+ \\
\sum_{n=1}^{N} \prod_{j=1}^{M_{\mathrm{M}}} \mathbb{1}_{\rho_{n}^{j}[k]=0} \mu_{n}^{m}[k] .
\end{array}
$$

where the indicator function $\mathbb{1}_{\{\rho\}}=1$ if and only if $\rho=0$ The SINR of the $n^{t h}$ MUE on subcarrier $k_{m}$ at the $m^{t h}$ FAP operating on mmWave band is given by

$$
\operatorname{SINR}_{n}^{m}\left[k_{m}\right]=\frac{\mu_{n}^{m}\left[k_{m}\right]}{\sigma^{2}\left[k_{m}\right]+I_{n}^{m}\left[k_{m}\right]},
$$

where $I_{n}^{m}\left[k_{m}\right]$ is defined by

$$
\begin{array}{r}
I_{n}^{m}\left[k_{m}\right]=\sum_{\substack{j=1 \\
j \neq m}}^{M} \sum_{i=1}^{F}\left(1-\prod_{\substack{a=1 \\
a \neq m}}^{M_{\mathrm{M}}} \mathbb{1}_{\left.\rho_{i_{j}}^{a}\left[k_{m}\right]=0\right) \mu_{f}^{m}\left[k_{m}\right]+}^{N}\left(1-\prod_{\substack{j=1 \\
j \neq m}}^{M_{\mathrm{M}}} \mathbb{1}_{\rho_{n}^{j}\left[k_{m}\right]=0}\right) \mu_{n}^{m}\left[k_{m}\right],\right.
\end{array}
$$

and the SINR of the $n^{\text {th }}$ MUE on the $k^{\text {th }}$ subcarrier at the $m^{t h}$ FAP operating on UHF band is expressed as

$$
\operatorname{SINR}_{n}^{m}[k]=\frac{\mu_{n}^{m}[k]}{\sigma^{2}[k]+I_{n}^{m}[k]},
$$

where $I_{n}^{m}[k]$ is defined by

$$
\begin{aligned}
I_{n}^{m}[k]=\sum_{j=1}^{M} \sum_{i=1}^{F}(1- & \left.\prod_{a=1}^{M_{\mathrm{U}}} \mathbb{1}_{\rho_{i_{j}}^{a}[k]=0}\right) \mu_{f}^{m}[k]+ \\
& \sum_{\substack{i=1 \\
i \neq n}}^{N} \prod_{j=1}^{M_{\mathrm{M}}} \mathbb{1}_{\rho_{i}^{j}[k]=0} \mu_{n}^{m}[k] .
\end{aligned}
$$

The SINR of the $n^{\text {th }}$ MUE at MBS on subcarrier $l$ is given by

$$
\operatorname{SINR}_{n}^{b}[l]=\frac{\mu_{n}^{b}[l]}{\sigma^{2}[l]+I_{n}^{b}[l]}
$$

where $I_{n}^{b}[l]$ is defined by

$$
\begin{array}{r}
I_{n}^{b}[l]=\sum_{j=1}^{M} \sum_{i=1}^{F}\left(1-\prod_{a=1}^{M_{\mathrm{U}}} \mathbb{1}_{\rho_{i_{j}}^{a}[k]=0}\right) \mu_{f_{j}}^{b}[l]+ \\
\sum_{\substack{i=1 \\
i \neq n}}^{N} \prod_{j=1}^{M_{\mathrm{M}}} \mathbb{1}_{\rho_{i}^{j}[l]=0} \mu_{n}^{b}[l] .
\end{array}
$$

The transmission power of all the users is limited to a maximum threshold denoted by $P^{\max }$. Each link between the user and the base station causes individual circuit power. In macrocell, it is denoted by $P_{C \text { (MBS) }}$ and it is represented as $P_{C(m)}$ in the $m^{t h}$ femtocell where $P_{C(\mathrm{MBS})}=P_{C(m)}=P_{C}$. Thus, the total power can be written as

$$
P_{T}=\epsilon \sum_{j \in \mathbb{J}} \sum_{i \in \mathbb{V}} \sum_{x \in \mathcal{K}} \rho_{i}^{j}[x] p_{i}^{j}[x]+(N+F M) \times P_{C},
$$

where $\epsilon$ represents the inverse of power amplifier efficiency. The EE, in bits/sec/Watt, is the amount of energy required by the system to transmit data and is expressed as

$$
\eta_{\mathrm{EE}}=\max _{p_{i}^{j}} \frac{\sum_{j \in J} \sum_{i \in \mathbb{I}} \sum_{x \in \mathcal{K}} R_{i}^{j}[x]}{\epsilon \sum_{j \in \downarrow} \sum_{i \in \mathbb{D}} \sum_{x \in \mathcal{K}} \rho_{i}^{j}[x] p_{i}^{j}[x]+(N+F M) \times P_{C}} .
$$

\section{Problem Formulation}

In our proposed scheme, two games are played in a hierarchical order. In the first game, each FAP decides between mmWave and UHF frequency bands with the goal to optimise its data rate forming a non-cooperative game. In the start, all FAPs have open access policy which allows them to connect with the MUEs to reduce the interference and maximise their rates. Let the fraction of the band allocated by the $m^{t h}$ FAP to the $i^{t h}$ user is denoted by $\omega_{i, m}$. This frequency band assignment to the FUEs and the MUEs by the FAPs forms the strategy space of FAPs in this game. Here, $\boldsymbol{\omega}_{m}=$ $\left[\omega_{n_{1}, m_{u}}, \ldots, \omega_{n_{N}, m_{u}}, \omega_{n_{1}, m_{m}}, \ldots, \omega_{n_{N}, m_{m}}, \omega_{f_{1}, m_{u}}, \ldots, \omega_{f_{F}, m_{u}}\right.$, $\left.\omega_{f_{1}, m_{m}}, \ldots, \omega_{f_{F}, m_{m}}\right]^{T}$ is the strategy vector of $m^{t h}$ FAP where $m_{u}$ represents the $m^{t h}$ FAP operating on UHF band and $m_{m}$ represents the $m^{\text {th }}$ FAP operating on mmWave band. $\boldsymbol{\omega}_{-m}=\left[\boldsymbol{\omega}_{1}^{T}, . ., \boldsymbol{\omega}_{m-1}^{T}, \boldsymbol{\omega}_{m+1}^{T}, . ., \boldsymbol{\omega}_{M}^{T}\right]^{T}$ shows the strategy vector of the other FAPs and $[.]^{T}$ denotes the transpose operator. The utility function of the $m^{\text {th }}$ FAP is the sum-rate of the FUEs and the MUEs connected to it.

$$
\begin{aligned}
\tilde{U}_{m}\left(\boldsymbol{\omega}_{m}, \boldsymbol{\omega}_{-m}\right)= & \sum_{i=1}^{F} \omega_{f_{i}, m} \log \left(1+\operatorname{SINR}_{i}^{m}\right)+ \\
& \sum_{i=1}^{N} \omega_{n_{i}, m} \log \left(1+\operatorname{SINR}_{i}^{m}\right) .
\end{aligned}
$$

The strategy space in this game for the $m^{t} h$ FAP is expressed as

$$
\tilde{\chi}_{m}=\left\{\boldsymbol{\omega}_{\boldsymbol{m}} \in[0, B]^{N}: \sum_{i=1}^{N_{m} \cup F_{m}} \omega_{i}^{m}=B\right\} .
$$


The above constraint makes sure that frequency allocation is well defined by each FAP. The optimization problem for the $m^{\text {th }}$ FAP, given the strategy vectors of other FAPs, is defined as

$$
\max _{\boldsymbol{\omega}_{m} \in \tilde{\chi}_{m}}\left(\boldsymbol{\omega}_{m}, \boldsymbol{\omega}_{-m}\right)
$$

This non-cooperative game achieves convergence using the solution of pure strategy Nash equilibrium (PSNE). A player achieves Nash equilibrium when

$$
\tilde{U}_{m}\left(\boldsymbol{\omega}^{*}{ }_{m}, \boldsymbol{\omega}^{*}{ }_{-m}\right) \geq \tilde{U}_{m}\left(\boldsymbol{\omega}_{m}, \boldsymbol{\omega}^{*}{ }_{-m}\right) ; \forall \omega_{m} \in \tilde{\chi_{m}},
$$

where $\omega_{m}$ represents the strategy vector of the $m^{\text {th }}$ player and $U_{m}$ represents the utility function.

The second game incorporates the user association to maximise the sum-rate and energy efficiency of the network. In this game, users evaluate their connectivity with the goal of maximizing their rates without affecting the network performance. The single-objective optimization problem (SOP) for this game can be written as

$$
\begin{array}{ll} 
& \max _{p_{i}^{j}} \quad \eta_{\mathrm{EE}} \\
\text { s.t. } \quad & \sum_{j \in \downarrow} R_{i}^{j}[\omega] \geq R_{\min }, \quad \forall i, \\
& \sum_{j \in \downarrow} p_{i}^{j}[\omega] \leq P_{i}^{\max }, \quad \forall i, \\
& \sum_{j \in J} \sum_{i \in \mathbb{I}} g_{i j} p_{i}^{j}[\omega] \leq I[\omega], \quad \forall \omega,
\end{array}
$$

where the first constraint ensures the achieved rate of the user is at least as high as $R_{\min }$. Second and third constraints limit the maximum transmission power of the users to maximise EE. Here, we have replaced the subcarriers with the fraction of band, $\left(\omega_{i, j}\right)$, allocated to the $i^{t h}$ user by the $j^{t h} \mathrm{BS}$.

The objective function can then be expressed as

$$
\begin{aligned}
& U\left(\eta_{E E}\right)=\max _{p_{i}^{j}} \quad\left[\sum_{j \in \rrbracket} \sum_{i \in \mathbb{I}} R_{i}^{j}[\omega]-\eta_{\mathrm{EE}}\left(\epsilon \sum_{j \in \mathbb{J}} \sum_{i \in \mathbb{I}} p_{i}^{(j)}[\omega]\right.\right. \\
& \left.\left.+(N+F M) \times P_{C}\right)\right] \text {. }
\end{aligned}
$$

The solution is formulated as Lagrangian dual decomposition approach [12]. The Lagrangian function of the above equation can be written as

$$
\begin{aligned}
& L(p, \boldsymbol{\lambda}, \boldsymbol{\mu}, \boldsymbol{\nu})=\sum_{m_{\mathrm{J}} \in \mathbb{J}} \sum_{i \in \mathbb{I}} R_{i}^{j}[\omega]-\eta_{\mathrm{EE}}\left(\epsilon \sum_{j \in \rrbracket} \sum_{i \in \mathbb{I}} p_{i}^{j}[\omega]\right. \\
& \left.+(N+F M) \times P_{C}\right)+\sum_{i \in \mathbb{N}} \lambda_{i}\left(\sum_{j \in \rrbracket} R_{i}^{j}[\omega]-R_{\min }\right) \\
& +\sum_{i \in \mathbb{N}} \mu_{i}\left(P_{i}^{\max }-\sum_{j \in \rrbracket} p_{i}^{j}[\omega]\right)+\nu_{\omega}\left(I[\omega]-\sum_{j \in \rrbracket} \sum_{i \in \rrbracket}\right. \\
& \left.p_{i}^{j}[\omega] g_{i j}\right) \text {, }
\end{aligned}
$$

where $\boldsymbol{\lambda}=\left\{\lambda_{1}, \lambda_{2}, \ldots, \lambda_{N+F}\right\}, \boldsymbol{\mu}=\left\{\mu_{1}, \mu_{2}, \ldots, \mu_{N+F}\right\}$ and $\nu=\left\{\nu_{1}, \nu_{2}, \ldots, \nu_{N+F}\right\}$ are the Lagrange multiplier vectors associated with $R_{\min }$, optimal transmit power and cross-tier interference threshold constraints, respectively.

The Lagrangian dual function is

$$
\begin{gathered}
g(\boldsymbol{\lambda}, \boldsymbol{\mu}, \boldsymbol{\nu})=\max _{p_{i}^{j}} \quad L\left(p_{n}^{m}, \boldsymbol{\lambda}, \boldsymbol{\mu}, \boldsymbol{\nu}\right) . \\
g(\boldsymbol{\lambda}, \boldsymbol{\mu}, \boldsymbol{\nu})=g_{\omega}(\boldsymbol{\lambda}, \boldsymbol{\mu}, \boldsymbol{\nu})-\epsilon \eta(N+F M) P_{C}+\nu_{\omega} I[\omega]+ \\
\sum_{i \in \mathbb{I}} \mu_{i} P_{i}^{\max }-\sum_{i \in \mathbb{I}} \lambda_{i} R_{\min },
\end{gathered}
$$

where $g_{\omega}(\boldsymbol{\lambda}, \boldsymbol{\mu}, \boldsymbol{\nu})$ is defined as

$$
\begin{array}{r}
g_{\omega}(\boldsymbol{\lambda}, \boldsymbol{\mu}, \boldsymbol{\nu})=\max _{p_{i}^{j}} \quad\left[\sum_{j \in \mathbb{J}} \sum_{i \in \mathbb{I}} R_{i}^{j}[\omega]-\eta \epsilon \sum_{j \in \mathfrak{J}} \sum_{i \in \mathbb{I}} p_{i}^{j}[\omega]\right. \\
+\sum_{i \in \mathbb{I}} \sum_{j \in \mathbb{J}} \lambda_{i} R_{i}^{j}[\omega]-\sum_{i \in \mathbb{\Xi}} \sum_{j \in \mathbb{J}} \mu_{i} p_{i}^{j}[\omega] \\
\left.-\sum_{i \in \mathbb{I}} \sum_{j \in J} \nu_{w} p_{i}^{j}[\omega] g_{i j}\right] .
\end{array}
$$

$$
\begin{aligned}
g_{\omega}(\boldsymbol{\lambda}, \boldsymbol{\mu}, \boldsymbol{\nu})=\max _{p_{i}^{j}} & \left(\sum_{j \in \mathbb{J}} \sum_{i \in \mathbb{I}} B_{w} \log \left(1+\beta_{i}^{j} p_{i}^{j}[\omega]\right)\left[1+\lambda_{i}\right]\right. \\
& \left.-\sum_{j \in \mathbb{J}} \sum_{i \in \mathbb{I}}\left(\mu_{i}+\epsilon \eta_{\mathrm{EE}}+\nu_{w} g_{i j}\right) p_{i}^{j}[\omega]\right) .
\end{aligned}
$$

where $\beta_{i}^{j}$ represents channel-to-interference and noise ratio of the $i^{\text {th }}$ user connected to $j^{\text {th }} \mathrm{BS}$.

We have decomposed the above dual problem into a hierarchical framework of two sub-problems. The master subproblem uses sub-gradient method to update the Lagrangian multipliers whereas the slave sub-problem consisting of $K$ sub-problems solved in parallel is responsible for computing power for given values of $\eta_{E E}$ and Lagrange multipliers. The first derivative of (30) w.r.t $p_{i}^{j}[\omega]$ is

$$
\frac{\partial g_{\omega}(\boldsymbol{\lambda}, \boldsymbol{\mu}, \boldsymbol{\nu})}{\partial p_{i}^{j}[\omega]}=\frac{B_{w}\left[1+\lambda_{i}\right] \beta_{i}^{j} p_{i}^{j}[\omega]}{\ln 2\left(1+\beta_{i}^{j} p_{i}^{j}[\omega]\right)} .
$$

Now, by applying KKT conditions, we get

$$
\left.\frac{\partial g_{\omega}(\boldsymbol{\lambda}, \boldsymbol{\mu}, \boldsymbol{\nu})}{\partial p_{i}^{j}[\omega]}\right|_{p_{i}^{j}[\omega]=p_{i}^{j}[\omega]^{*}}=0
$$

Hence,

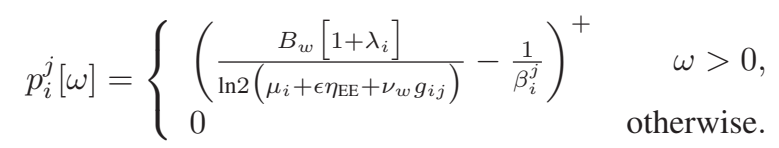

The optimal solution of (25) can be expressed as

$$
p_{i}^{j *}=\min \left(p_{i}^{j}[\omega], P_{i}^{\max }\right)
$$

Now, we can update the Lagrange multipliers as

$$
\lambda_{i}(k+1)=\left(\lambda_{i}(k)-\frac{\alpha^{1}}{\sqrt{k}}\left(\sum_{i \in \rrbracket} \sum_{j \in \rrbracket} R_{i}^{j}[\omega]-R_{\min }\right)\right)^{+},
$$




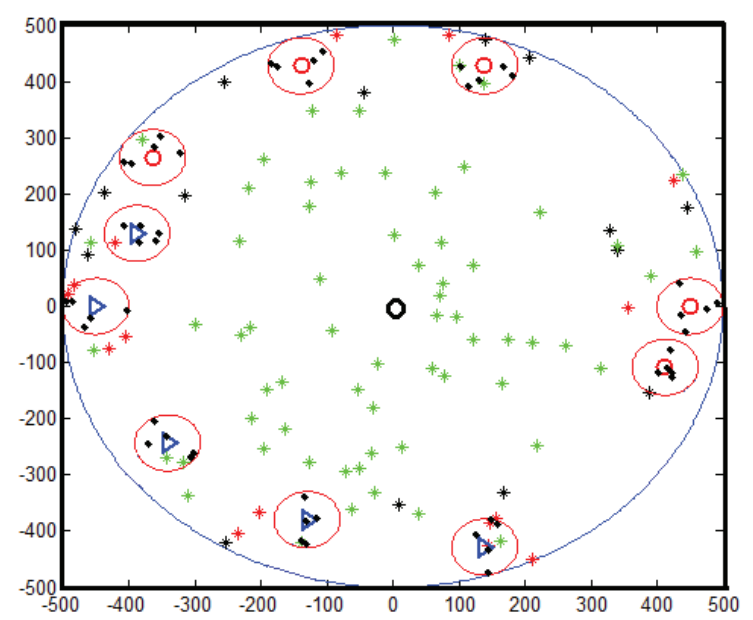

Figure 2. Snapshot of the HetNet with blue triangles showing UHF FAPs and red circles showing mmWave FAPs. Green stars representing the MUEs connected to MBS, red stars representing the MUEs connected to FAPs and black stars representing the MUEs in outage $(\mathrm{M}=10, \mathrm{~N}=100$ and $\mathrm{F}=5)$.

$$
\begin{aligned}
& \mu_{i}(k+1)=\left(\mu_{i}(k)-\frac{\alpha^{2}}{\sqrt{k}}\left(P_{i}^{\max }-\sum_{i \in \mathbb{I}} \sum_{j \in \mathbb{J}} p_{i}^{j}[\omega]\right)\right)^{+}, \\
& \nu_{\omega}(k+1)=\left(\nu_{\omega}(k)-\frac{\alpha^{3}}{\sqrt{k}}\left(I[\omega]-\sum_{i \in \mathbb{I}} \sum_{j \in \rrbracket} p_{i}^{j}[\omega] g_{i j}\right)\right)^{+} .
\end{aligned}
$$

where $\alpha$ is the step length and $i$ is the iteration number. These equations continues to update until convergence is achieved.

\section{Simulation Results}

We consider a two-tier HetNet with a single macrocell of radius $500 \mathrm{~m}$ where femtocells with the radius of $50 \mathrm{~m}$ each are uniformly overlaid on it as shown in Fig. 2. The users are also uniformly scattered over the area. The number of FUEs serviced by each FAP are 5 unless mentioned otherwise. The system bandwidth, $B$, for UHF band is $20 \mathrm{MHz}$ and for mmWave band the bandwidth, $B_{\mathrm{m}}$, is $2 \mathrm{GHz}$ [6]. The maximum transmission power $P_{\max }$ is set to be $0.4 \mathrm{~W}$ and the minimum acceptable data rate for the MUEs, $R_{\min }$, is 0.25 Mbps. These thresholds are same for all the users. The value of circuit power is fixed to be $0.1 \mathrm{~W}$, power amplifier efficiency $\epsilon$ is $38 \%$ and the interference threshold is $1.1943 \times 10^{-14} \mathrm{~W}$ unless stated otherwise. The parameters for path loss models are listed in Table. I.

Table I

Simulation PARAMETERS.

\begin{tabular}{|c|c|c|c|}
\hline Parameter & Value & Parameter & Value \\
\hline$f_{c}(\mathrm{mmW})$ & $73 \mathrm{GHz}$ & $f_{c}(\mathrm{UHF})$ & $2.4 \mathrm{GHz}$ \\
\hline$\alpha_{L}$ & 2.2 & $\alpha_{N}$ & 3.3 \\
\hline$\sigma_{\Omega_{L}}$ & $5.2 \mathrm{~dB}$ & $\sigma_{\Omega_{N}}$ & $7.38 \mathrm{~dB}$ \\
\hline$\sigma_{\Psi}$ & $4 \mathrm{~dB}$ & $\begin{array}{c}\text { K-factor } \\
\text { (Rician) }\end{array}$ & $4 \mathrm{~dB}$ \\
\hline
\end{tabular}

We have analysed the sum-rate and energy efficiency of the proposed hybrid HetNet and all-UHF HetNet with and

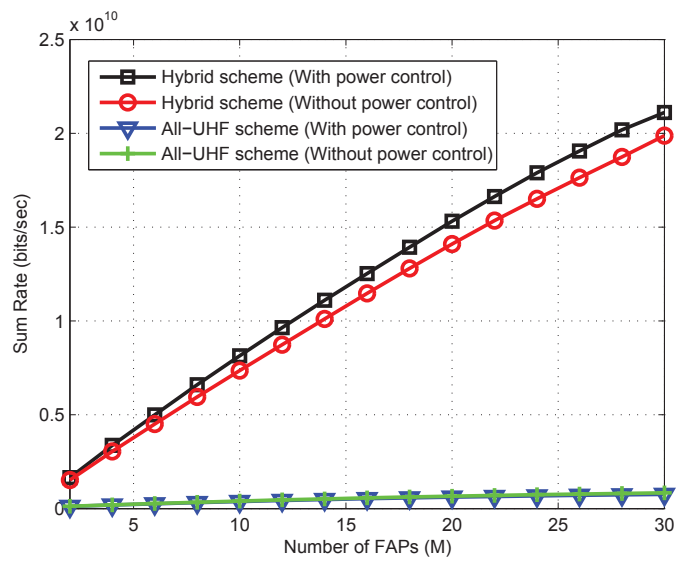

Figure 3. Sum-rate of a hybrid HetNet and all-UHF HetNet with and without power control with varying number of FAPs for $\mathrm{N}=100$ and $\mathrm{F}=5$.

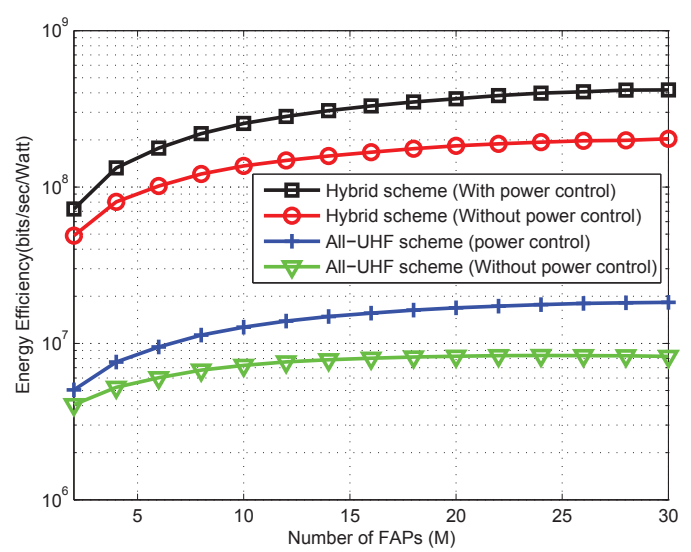

Figure 4. Energy Efficiency of a hybrid HetNet and all-UHF HetNet with and without power control with varying number of FAPs for $\mathrm{N}=100$ and $\mathrm{F}=5$.

without power control mechanism. This proposed network allows FAPs to decide their access policy in the best interest of their users and MUEs to finalize their connectivity to maximise the energy efficiency while fulfilling all the constraints. This hybrid scheme outperforms the all-UHF scheme as shown in Fig. 3 and Fig. 4 because the UHF network shows better coverage probabilities at lower SINR thresholds as they provide higher SINR at the base station for the cell edge users. The mmWave network, on the other hand, provides better coverage when the users are located near the base station as it undergoes lower interference from the neighbouring users. Thus, a fusion of both networks leads to better performance. An increasing trend is shown in all schemes in the sum-rate and energy efficiency with increasing number of FAPs. This is due to the fact that as the FAPs increases, they connect more MUEs and thus reduce the interference in the network. The performance of this hybrid scheme further improves when power control is applied. By limiting transmission power to the optimal value, the cross-tier interference reduces, which in return increases the SINR; thus improving the sum-rate and the energy efficiency.

Fig. 5 reveals that the energy efficiency of a hybrid heterogeneous network increases as the value of interference 


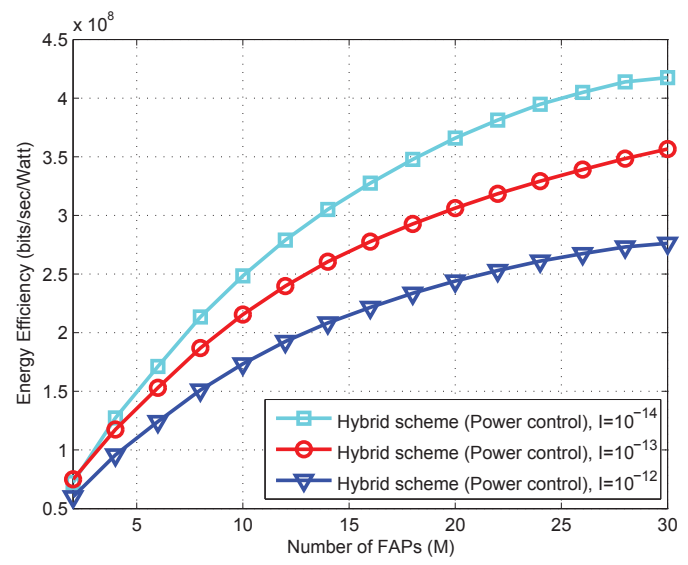

Figure 5. Energy Efficiency of a hybrid HetNet with power control for various interference threshold with varying number of FAPs for $\mathrm{N}=100$ and $\mathrm{F}=5$.

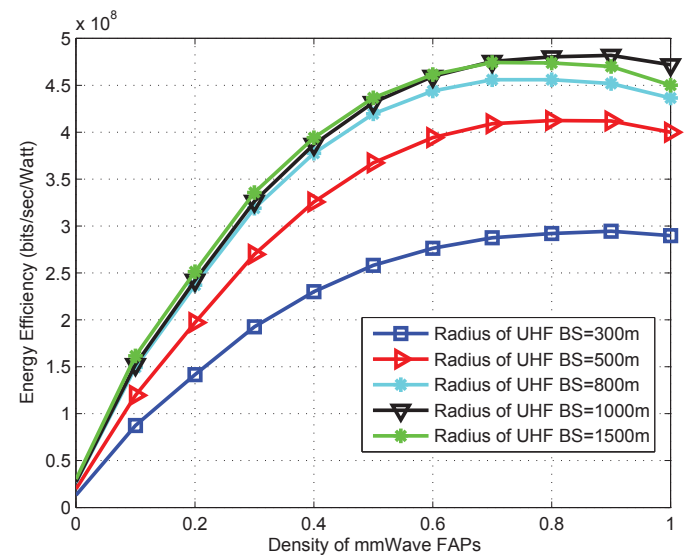

Figure 6. Energy Efficiency of a hybrid HetNet with power control with varying density of mmWave FAPs for $\mathrm{M}=15, \mathrm{~F}=5$ and $\mathrm{N}=100$.

threshold decreases. The trend shows that as the threshold level decreases, the corresponding transmit power of the users decreases, which results in the reduction of interference. This reduction in the interference leads to the increment in the SINR; thus improving the sum-rate and the energy efficiency.

Fig. 6 shows that the trend of EE associated with the density of mmWave FAPs. We can observe that the EE is very low when the density of mmWave FAPs is zero i.e. all UHF scheme. As the density of FAPs operating on mmWave increases, the users located near the FAPs will get better coverage and thus data rates and EE increases. This trend becomes steady after a while as the FAPs serving the MUEs start dominating. This is due of the fact that the mmWave FAPs restrict their ability to form links over long distances due to greater path loss associated with mmWave and it is in the best interest of the network that these FAPs should operate on UHF band. Thus, a hybrid approach offers better data rates and EE than all-UHF and all-mmWave femto-tier network. From the figure, we can also observe that as the radius of the network increases, the distance between the MUEs and the FAPs increases, which will reduce the interference. Thus relatively less MUEs connect with the FAPs and the near located users play the major role making more FAPs to operate on mmWave due to better coverage. This trend follows up to a certain radius of the network, then it starts decreasing if we further increase the radius as the SINR start decreasing.

\section{CONCLUSION}

In this paper, a hierarchical game-theoretic framework is formulated to optimise the energy efficiency in a two-tier hybrid HetNet while incorporating maximum transmit power and interference constraint. This scheme allows FAPs to decide their access policy along with the selection of frequency band in between sub- $6 \mathrm{GHz}$ and mmWave. The user association method is then carried out such that the energy efficiency is maximised. The proposed game framework is solved using PSNE for the outer layer and Lagrangian dual decomposition approach for the inner layer. Simulation results show that in contrast to the all-UHF network, hybrid networks promise performance enhancement in terms of energy efficiency. The performance of the proposed design can be further improved using power control mechanism that aims at limiting the interference and increasing the SINR.

\section{REFERENCES}

[1] Q. Ye, B. Rong, Y. Chen, M. Al-Shalash, C. Caramanis, and J. G. Andrews, "User association for load balancing in heterogeneous cellular networks," IEEE Trans. on Wireless Commun., vol. 12, no. 6, pp. 2706-2716, 2013

[2] C. S. Chen, F. Baccelli, and L. Roullet, "Joint optimization of radio resources in small and macro cell networks," IEEE Vehicu. Tech. Conf. (VTC-Spring), pp. 1-5, 2011

[3] A. U. Rahman and S. A. Hassan, "Analysis of composite fading in a single cell downlink cooperative heterogeneous networks," IEEE Vehicu. Tech. Conf. (VTC-Spring), pp. 1-5, 2015.

[4] T. S. Rappaport, R. W. Heath Jr., R. C. Daniels, J. N. Murdock, Millimeter Wave Wireless Communication, Prentice Hall, 2014.

[5] A. Ghosh, T. A. Thomas, M. C. Cudak, R. Ratasuk, P. Moorut, F. W. Vook, T. S. Rappaport, G. R. MacCartney, S. Sun, S. Nie, "Millimeter wave enhanced local area systems: A high data rate approach for future wireless networks," IEEE J. Sel. Areas Commun., vol. 32, no. 6, pp.1152-1163, Jun. 2014.

[6] S. Singh, M. N. Kulkarni, A. Ghosh, and J. G. Andrews "Tractable model for rate in self-backhauled millimeter wave cellular networks", IEEE J. Sel. Areas Commun., vol. 33, no. 10, pp. 2196-2211, 2015.

[7] S. Rangan, T. S. Rappaport, and E. Erkip, "Millimeter-wave cellular wireless networks: Potentials and challenges", IEEE Proceedings, vol. 102, no. 3, pp. 366-385, 2014.

[8] S. E. Elayoubi, E. Altman, M. Haddad, and Z. Altman, "A hybrid decision approach for the association problem in heterogeneous networks," IEEE INFOCOM, pp. 1-5, 2010.

[9] R. Hu and Y. Qian, "An energy efficient and spectrum efficient wireless heterogeneous network framework for 5G systems", IEEE Commun. Mag., vol. 52, no. 5, pp. 94-101, 2014.

[10] H. Pervaiz, L. Musavian, and Q. Ni, "Area energy and area spectrum efficiency trade-off in 5G heterogeneous networks," IEEE International Conf. on Commun. Workshop (ICCW), pp. 1178-1183, 2015.

[11] J. Liu, D. Wang, J. Wang, J. Li, J. Pang, G. Shen, Q. Jiang, H. Sun, and Y. Meng, "Uplink power control and interference coordination for heterogeneous network,'IEEE International Symposium on Personal Indoor and Mobile Radio Communications (PIMRC), pp. 519-523, 2012.

[12] D. P. Palomar and M. Chiang, "A tutorial on decomposition methods for network utility maximization," IEEE Journal on Selected Areas in Commun., vol. 24, no. 8, pp. 1439-1451, 2006. 